\title{
Inter-relação entre aptidão física relacionada a saúde e domínios da Qualidade de vida
}

\section{Bruno Pedroso}

prof.brunopedroso@gmail.com

Universidede Gonta (UEPG), Ponta Grossa, Paraná, Brasil

\section{Guilherme Moreira Caetano Pinto} prof.guilhermecaetano@gmail.com Universidade Estadual de Ponta Grossa (UEPG), Ponta Grossa, Paraná, Brasil

José Roberto Herrera Cantorani cantorani@yahoo.com.br

Instituto Federal de Educação (IFE), Registro, São Paulo, Brasil

\section{Claudia Tania Picinin}

claudiapicinin@utfpr.edu.br

Universidade Tecnológica Federal do Paraná (UTFPR), Ponta Grossa, Paraná, Brasil

Luiz Alberto Pilatti

lapilatti@utfpr.edu.br

lapilatti@utfpr.edu.br

Paraná (UTFOR), Ponta Grossa, Paraná,

Brasil
Este estudo almeja verificar a correlação entre aspectos da aptidão física relacionada à saúde com domínios da qualidade de vida (QV) de estudantes de ensino superior, participantes do projeto de extensão UTFPR em Ação. Para a avaliação da QV aplicou-se o instrumento WHOQOL-bref. Para a avaliação da aptidão física utilizou-se do índice de Massa Corpórea (IMC), percentual de gordura e os testes: Banco de Wells, Cooper, abdominais e flexão de braços no solo por minuto (RML). Os resultados retornaram correlações significativas entre os dados dos testes de RML e de Cooper com os escores do domínio físico do WHOQOL-bref. Os resultados dos testes RML, de Cooper, IMC e percentual de gordura com os domínios psicológico, ambiente, relações sociais do WHOQOL-bref não se correlacionaram significativamente. Conclui-se que, quanto maiores forem os índices de RML e resistência aeróbica, maior será a satisfação do indivíduo em relação ao domínio físico. Tal correlação não ocorreu em outros cenários investigados neste estudo.

PALAVRAS-CHAVE: Aptidão física; Saúde; Qualidade de vida. 


\section{INTRODUÇÃO}

A qualidade de vida (QV) é uma temática bastante abarcada por diversas áreas acadêmicas, com notável destaque. Ainda antes de se tornar um objeto de estudo, o termo QV foi utilizado em um discurso político com o intuito valorizar a gestão pública para além de fatores econômicos. Em 1964 o então presidente americano Lyndon Johson defendeu seu governo afirmando que "os objetivos não podem ser medidos através do balanço dos bancos. Eles só podem ser medidos através da QV que proporcionam às pessoas" (FLECK et al., 1999, p. 19).

Alheio ao viés político, durante anos o meio acadêmico destinou esforços para conceituar a QV, produzir instrumentos de avaliação e mensurar a QV em diversos públicos.

Em relação ao conceito, ainda que os esforços tenham contribuído para uma melhor compreensão acerca do significado do termo, e que a QV apresente um evidente profusão teórica, é prudente ressaltar que não é possível conceituar a QV em um único e absoluto conceito (PEDROSO; PILATTI, 2012; OLIVEIRA, 2017).

A QV, conceitualmente, apresenta distintas vertentes em função da diversidade de áreas que a pesquisam e avaliam. No presente estudo entende-se a QV como "a percepção do indivíduo de sua posição na vida no contexto da cultura e sistema de valores nos quais ele vive e em relação aos seus objetivos, expectativas, padrões e preocupações" (FLECK, 2000, p. 34).

A evolução conceitual do termo possibilitou diferentes encaminhamentos de pesquisas. A origem das pesquisas na área é clínica, e, neste viés, justificam-se na possibilidade de, antes, durante ou após um tratamento, mensurar a QV para verificar a eficácia de uma intervenção clínica efetuada (PATRICK, 2008). Não obstante, a avaliação da QV é também direcionada à detecção de pontos de carência de um grupo populacional e/ou para distinguir fatores externos que influenciam a QV do indivíduo. Tais possibilidades são essenciais para se fundamentar a tomada de decisões de estratégias que visem melhorar a QV de um grupo.

Em relação às metodologias, a literatura dispõe de inúmeras formas de avaliação da QV, sem definição de um "padrão-ouro" (FARQHAR, 1995). De maneira geral, a entrevista e a aplicação de questionários são as metodologias mais comuns encontradas para avaliar a QV nos mais diferentes grupos populacionais. É possível afirmar que não há diferenças significativas nos resultados obtidos na avaliação de QV quando empregadas essas duas formas de pesquisa (SEIDL; ZANNON, 2004).

Entre os fatores que se relacionam à qualidade de vida, elenca-se a saúde, os hábitos de vida, as condições de vida, as escolhas nutricionais, o nível de atividade física, e o uso de drogas (VEIGA; CANTORANI; VARGAS, 2016; PEREIRA et al., 2009). Por conseguinte, este artigo baseia-se em constructos teóricos que indicam alto grau de associação entre às variáveis saúde, aptidão física relacionada à saúde (AFRS) e QV, bem como o consenso de que o domínio "saúde" exerce forte influência sobre a QV (ARAÚJO; ARAÚJO, 2000; MAZO et al., 2009).

No tocante à AFRS, a literatura científica aponta para o entendimento de que o risco de aparecimento de doenças hipocinéticas ou crônico-degenerativas é maior quando os índices das capacidades físicas cardiorrespiratória, de resistência muscular, flexibilidade, e os níveis de gordura corporal estão inadequados (GLANER, 2005; PRACIDELI; CABRAL, 2011; US DEPARTAMENT OF HEALTH AND HUMAN SERVICES, 1996). 
Neste sentido, cabe ressaltar que o exercício físico, capaz de melhorar os níveis de AFRS, também é considerado um importante aliado no combate às doenças crônicas adquiridas por hábitos de vida não ativos fisicamente (ALONSO et al., 2005). Não obstante, estudos apontam que a realização de exercícios físicos, exercícios aeróbicos, e exercícios físicos direcionados à flexibilidade impactam positivamente na percepção da QV de adultos ou idosos (AMORM; DANTAS, 2002; PRACIDELI; CABRAL, 2011). Tais dados argumentam em favor da hipótese de que indivíduos com altos índices de AFRS tendem a apresentar um maior escore da QV geral e de seus domínios.

O que aqui se apresenta é um ideário de correção entre Saúde, AFRS e QV. Cabe argumentar, no entanto, que o meio acadêmico não dispõe de pesquisas que busquem correlacionar as variáveis ligadas à AFRS com os domínios pertinentes à QV. Frente a este cenário e a esta problemática, o presente estudo tem por objetivo verificar a correlação entre os resultados dos testes da AFRS e os escores de QV de estudantes de ensino superior participantes de um projeto de extensão voltado para a prática de atividades físicas no intento da melhoria da QV, o projeto UTFPR em Ação.

\section{PROCEDIMENTOS METODOLÓGICOS}

Para a obtenção da amostra utilizou-se o método de amostragem não probabilística por acessibilidade. A amostra totalizou 75 estudantes universitários de da UTFPR - Câmpus Ponta Grossa. Destes, 20 eram do gênero feminino e 55 do gênero masculino.

Para a avaliação da QV foi utilizado o instrumento WHOQOL-bref, composto por 26 questões (PEDROSO, 2013). Desse total de questões, duas estão relacionadas à auto avaliação da QV, e 24 dizem respeito aos seguintes domínios da vida: físico, psicológico, relações sociais e meio ambiente (CHACHAMOVICH; FLECK, 2008).

Para a avaliação da AFRS utilizou-se os seguintes testes: do Banco de Wells, para avaliação da Flexibilidade; de abdominais por um minuto (RML-1) e flexão de braços no solo por um minuto (RML-2), para mensurar a RML; e de Cooper, para avaliar a capacidade aeróbica. Para a composição corporal foram realizados o cálculo de IMC, por meio das variáveis peso e altura, e também a avaliação do percentual de gordura, efetuada por meio da balança digital com bioimpedância da marca Tanita, modelo BC-533 InnerScan Body Composition Monitor.

Para a análise dos dados retornados pelo instrumento WHOQOL-bref utilizou-se a ferramenta para cálculo dos escores e estatística disponibilizada em Pedroso et al. (2010). A referida ferramenta trata-se de uma planilha desenvolvida no software Microsoft Excel e efetua os cálculos de forma automática, cabendo ao pesquisador efetuar apenas a tabulação dos dados. Após a tabulação dos dados, a planilha apresenta gráficos para a apresentação de resultados, bem como tabelas com a estatística descritiva (PEDROSO et al., 2010).

A classificação dos resultados para os testes de RML-1 e RML-2 pautou-se na proposta de Pollock e Wilmore apud Hoffman (2006). Já os resultados obtidos no teste de flexibilidade foram classificados de acordo com a proposta do Canadian Standardized Teste of Fitness (CSTF) (CSTF apud RIBEIRO et al., 2010). Optou-se por esta proposta devido ao fato de que o CSTF, assim como na proposta de Pollock e Wilmore apud Hoffman ${ }^{16}$ para os testes de RML-1 e RML-2, considera valores de referência distintos para grupos do gênero masculino e feminino. Os 
resultados do teste de Cooper foram calculados através da fórmula VO2 MÁX $(\mathrm{ml} / \mathrm{kg} / \mathrm{min})=22,351 \mathrm{X}$ distância (KM) -11,288 (GARCIA, 2009).

Os resultados de IMC foram classificados de acordo com a proposta da Organização Mundial da Saúde (OMS) para indivíduos adultos (OMS, 2000). O percentual de gordura foi classificado de acordo com a proposta de Pollock e Wilmore apud Hoffman (2006).

O cálculo de correlação entre os resultados dos testes da AFRS e dos domínios da QV foi efetuado por meio da correlação de Pearson. Os critérios de exclusão do presente estudo foram: Preenchimento inferior a $80 \%$ do instrumento WHOQOL-Bref; Não cumprir o jejum na avaliação de composição corporal; Não conclusão de $50 \%$ dos testes físicos da AFRS.

O programa utilizado para o cálculo estatístico da amostra foi software o Statistical Package for the Social Sciences - IBM SPSS Statistics for Windows, versão 23.0. Para averiguar a normalidade dos dados foi utilizado o teste de Kolmogorov-Smirnov, e o teste utilizado para verificar a relação entre as variáveis foi realizado o reste de correlação de Pearson.

Os resultados dos cálculos propostos foram analisados em relação às hipóteses do modelo de análise, de acordo com a literatura de Dancey e Reidy (2006).

\section{RESULTADOS E DISCUSSÃO}

Para facilitar as análises das correlações efetuadas, os resultados foram organizados em dois grupos: composição corporal e QV, que compreende a correlação de IMC e Percentual de gordura com os domínios do WHOQOL-bref; e condicionamento físico e QV, que engloba a correlação entre os testes de resistência muscular localizada, flexibilidade e resistência aeróbica com os domínios do WHOQOL-bref.

A tabela 1 apresenta os resultados da avaliação de IMC da presente amostra, considerando a classificação proposta pela OMS para indivíduos adultos (OMS, 2000).

Tabela 1 - IMC Retornado pela amostra de acordo com a classificação proposta pela OMS

$\begin{array}{cc}\text { Baixo Peso (IMC < 18,5) } & 9,34 \% \\ \text { Peso normal (IMC 18,5 a 24,9) } & 72 \% \\ \text { Sobrepeso (IMC } \geq 25) & - \\ \text { Pré-obeso (IMC 25,0 a 29,99) } & 12 \% \\ \text { Obeso I (IMC 30,0 a 34,99) } & 2,66 \% \\ \text { Obeso II (IMC 35,0 a 39,99) } & 4 \% \\ \text { Obeso III (IMC > 40,0) } & -\end{array}$

Fonte: Pesquisa de Campo (2015).

Observa-se que a amostra investigada apresenta predomínio para a classificação Peso normal (72\%). Diante deste resultado, verifica-se, no que concerne à avaliação de IMC, que a amostra apresentou tendência homogênea. Tais resultados assemelham-se ao reportado por Cruz et al., (2018), que investigou o estado nutricional de estudantes de nutrição de uma faculdade de 
Bauru-SP e encontrou que $71 \%$ dos alunos encontravam peso normal. Em medida semelhante, Fernani et al. (2017) mostraram que 67\% dos universitários da área da saúde encontram-se no peso ideal, e $20 \%$ encontravam-se com sobrepeso.

Cabe ressaltar que o excesso de peso da população é considerado um problema mundial que se estende a distintas faixas etárias (CASTRO et al., 2018). Neste sentido, ainda que grande parte da população universitária investigada neste estudo esteja no peso normal (72\%), $18,66 \%$ da população encontram-se com sobrepeso ou obesidade. O cenário ilustrado somada a preocupação mundial com o excesso de peso indica que este problema requer atenção acadêmica e social.

A tabela 2 apresenta os resultados da avaliação de percentual de gordura, considerando a classificação de Pollock e Wilmore apud Hoffman (2006).

Tabela 2 - Percentual de gordura retornado pela amostra de acordo com a classificação proposta por Pollock e Wilmore apud Hoffman (2006)

\begin{tabular}{cc} 
Idade & Percentual de gordura \\
Acima da média & $21,4 \%$ \\
Abaixo da média & $20 \%$ \\
Bom & $18,6 \%$ \\
Média & $16 \%$ \\
Excelente & $12 \%$ \\
Ruim & $9,34 \%$ \\
Muito Ruim & $2,66 \%$ \\
\hline
\end{tabular}

Fonte: Pesquisa de Campo (2015).

Verifica-se, ao comparar os resultados do teste de IMC com o teste de percentual de gordura, que os resultados se assemelham visto que há proximidade na representação percentual da amostra entre os indivíduos que apresentam um IMC classificado como peso normal (72\%) e aqueles com percentual de gordura entre a classificação média ou superiores (68\%).

No que tange ao percentual de gordura, Mazini Filho et al. (2015) reportaram que $57,4 \%$ dos acadêmicos universitários de uma instituição de minas gerais apresentaram percentual de gordura adequado. No presente estudo, considerando os indivíduos que reportaram um percentual de gordura acima da média ou superior, o percentual retornado é de $62 \%$, indicando que estes valores são comuns a públicos universitários.

Quanto aos testes físicos, à tabela 3 apresenta o nível de aptidão física para os testes de RML-1, RML-2 e flexibilidade. Ressalta-se que a classificação acima da média tem conotação positiva, visto que indica que o resultado obtido pelo indivíduo foi superior à média populacional. Em medida semelhante, a classificação abaixo da média tem conotação negativa, e indica que o resultado foi inferior à média populacional. 
Tabela 3 - Nível de aptidão física dos testes das capacidades físicas relacionadas à saúde

\begin{tabular}{cccc|}
\hline Classificação & Abdominal & Flexão de Braços & Flexibilidade \\
Excelente & $6,66 \%$ & $9,34 \%$ & $13,33 \%$ \\
Acima da média & $13,34 \%$ & $22,66 \%$ & $13,34 \%$ \\
Média & $18,66 \%$ & $22,66 \%$ & $22,67 \%$ \\
Abaixo da média & $33,34 \%$ & $21,34 \%$ & $20 \%$ \\
Fraco & $28 \%$ & $24 \%$ & $30,66 \%$ \\
\hline
\end{tabular}

Fonte: Pesquisa de Campo (2015).

No teste de RML-1, houve um predomínio de indivíduos nas classificações Fraco ou Abaixo da média, que atingiu 61,34\% dos participantes. No teste de RML-2 a soma das classificações Fraco ou Abaixo da média atingiu 45,34\%. O teste de flexibilidade reforça a baixa aptidão física dos acadêmicos investigados. As classificações Fraco e Abaixo da média somam juntas $50,66 \%$ dos participantes.

No tocante às variáveis expostas na tabela 3 Mazini Filho et al. (2015) reportaram que 51,9\% apresentaram nível de flexibilidade adequado e $81,4 \%$ apresentaram nível de resistência muscular localizada adequado, considerando acadêmicos universitários de uma instituição de minas gerais apresentaram percentual de gordura adequado.

No presente estudo, considerando a classificação acima da média ou superior, $20 \%$ apresentaram bom nível no teste de abdominal, 32\% apresentaram bom nível no teste de flexão de braços, e $26,67 \%$ apresentaram bom nível de flexibilidade.

Ao efetuar a comparação destes valores observa-se uma disparidade, na qual fica evidenciada uma maior aptidão física na amostra de Mazini Filho et al. (2015). No entanto, é prudente mencionar que o referido estudo trabalhou com estudantes do curso de Educação Física, que naturalmente tem uma ligação maior com exercício físico. Este fato pode justificar este cenário.

Em relação à capacidade aeróbica, a tabela 4 apresenta a classificação retornada para esta amostra no teste de Cooper.

Tabela 4 - Capacidade aeróbica avaliada pelo teste de cooper calculada pelo portal The Global Fitness Community

$\begin{array}{cc}\text { Classificação } & \text { Capacidade Aeróbica } \\ \text { Fraco } & 93,4 \% \\ \text { Regular } & 4 \% \\ \text { Boa } & 2,6 \%\end{array}$

Fonte: Pesquisa de Campo (2015).

Verifica-se que quase a totalidade dos indivíduos investigados apresentaram uma resistência aeróbica insatisfatória. A amostra, quanto a esta variável, apresentou tendência homogênea.

No tocante à capacidade aeróbicas Mazini Filho et al. (2015) reportaram que $55,6 \%$ encontram-se em boa resistência cardiorrespiratória, considerando acadêmicos universitários de uma instituição de minas gerais apresentaram percentual de gordura adequado.

Tal qual no exposto relacionado a RML e flexibilidade, ao observar os resultados do presente estudo verifica-se que apenas $2,6 \%$ apresentaram boa 
capacidade aeróbica, valor muito inferior aos 55,6\% mencionados por Mazini Filho et al. (2015). Neste sentido, parece que a natureza do curso também justifica este cenário.

A tabela 5 apresenta os valores de correlação entre a composição corporal (IMC e percentual de gordura) e QV (domínios do WHOQOL-bref, faceta de auto avaliação da QV e escore Geral).

Tabela 5 - Correlação de Pearson das variáveis da composição corporal IMC e percentual de gordura com os domínios retornados pelo instrumento WHOQOL-Bref

\begin{tabular}{ccc} 
Domínio & IMC & $\begin{array}{c}\text { Percentual de } \\
\text { Gordura }\end{array}$ \\
Domínio físico & 0,138 & $-0,073$ \\
Domínio psicológico & 0,126 & $-0,091$ \\
Domínio relações sociais & 0,052 & $-0,005$ \\
Domínio ambiente & 0,051 & $-0,180$ \\
Auto avaliação da QV & $-0,151$ & $-0,129$ \\
Geral & 0,091 & $-0,129$ \\
\hline
\end{tabular}

*Correlações significativas no nível $p>0,05$ quando valores forem maiores que 0,227 ou menores que $-0,227$. Correlações significativas no nível $p<0,05$ quando valores forem maiores que 0,296 ou menores que -0,296.

Fonte: Pesquisa de campo (2015).

Verifica-se, face ao exposto, que as correlações não foram significativas para $p>0,05$ e p>0,01 entre os domínios do instrumento WHOQOL-bref e o IMC e percentual de gordura da população em exame. Para a presente amostra não foi verificado correlação do IMC e do percentual de gordura com os domínios físico $(0,138 /-0,073)$, psicológico $(0,126 /-0,091)$ e relações sociais $(0,052 /-0,005)$.

Garcia, Moretto e Guariento (2016) evidenciaram que problemas como a desnutrição e a obesidade estão relacionados com uma pior QV. Por outro lado, a relação entre a composição corporal e a QV torna-se contraditória na literatura. Para exemplificar tal afirmação arrolou-se dois estudos direcionados a população de idosos. Rodrigues, Abourihan e Yamane (2010) indicaram, em idosos, que os indivíduos com sobrepeso apresentaram uma melhor percepção sobre sua QV. Por sua vez, Adami, Feil e Bosco (2015) indicaram que quanto maior foi o IMC de idosos, pior foi sua $\mathrm{QV}$ relacionada ao domínio Físico.

Neste sentido, os resultados da presente pesquisa seriam um indicativo de que não se confirma a hipótese de que a composição corporal influenciaria a satisfação com o bem-estar físico, ou mesmo afetaria a interação social ou geraria desordens emocionais. No entanto, a homogeneidade da amostra, com tendência para Peso normal no IMC, e, para uma boa condição no percentual de gordura, não permitem tal inferência. Neste caso, o " $n$ " reduzido, somado à referida homogeneidade, configuram um fator de limitação do estudo. Também não foram encontradas correlações significativas entre a autoavaliação da QV ($0,151)$ e o domínio geral $(0,091)$ com o IMC. A estes dados, o mesmo fator limitante se aplica.

Conte et al. (2003), ao verificar as interseções entre as variáveis IMC e o VO ${ }^{2}$ máx., IMC e flexibilidade, e VO2 máx. e flexibilidade em indivíduos adultos afirma que, tanto para homens quanto para mulheres, há uma chance elevada de que indivíduos com alto desempenho em testes de $\mathrm{VO}^{2}$ máx. apresentem o IMC e a Flexibilidade dentro dos valores adequados, bem como de que indivíduos com 
baixo rendimento em testes de $\mathrm{VO}^{2}$ máx. apresentem o IMC elevado e a flexibilidade com valores fora do padrão adequado. Com base nestes resultados, Conte et al. (2003) infere que o controle da composição corporal é fundamental para a saúde e a QV.

Porém, em que pese os resultados apresentados por Conte et al. (2003), não foi possível observar nenhuma correlação entre as variáveis da composição corporal e a auto percepção da QV da população investigada.

A limitação do presente estudo, aqui já referida, pode justificar o cenário acima exposto: os dados da classificação de IMC retornaram que $72 \%$ dos indivíduos analisados estavam na classificação de eutróficos, havendo notaria disparidade com as classificações de abaixo do peso $(9,34 \%)$, sobrepeso $(12 \%)$, obesidade $1(2,66 \%)$ e obesidade 2 (4\%); o mesmo ocorre em relação à classificação do percentual de gordura, para a qual os dados mostram que 53,33\% dos indivíduos avaliados apresentaram classificação entre Excelente, Acima da média ou Bom. Apenas $16 \%$ da presente amostra apresentaram classificação média e, por fim, 30,67\% dos indivíduos avaliados apresentaram às classificações Abaixo da média, Ruim ou Muito ruim.

Dessa forma, admite-se que a característica homogênea da amostra nas variáveis de IMC e percentual de gordura dificultaram a verificação de correlações entre estas variáveis e os domínios da QV. Diante de tal contexto é verificada a necessidade de ampliação da amostra para um estudo dessa natureza. Não obstante, frente ao fato de que a QV é um conceito fortemente ligado à saúde, amplia-se o entendimento de que é justificável a ampliação de estudos a este respeito.

A tabela 6 apresenta a correlação de Pearson das variáveis relacionadas à resistência muscular localizada nos testes de RML-1 e RML-2, flexibilidade e resistência aeróbica com os domínios presentes no instrumento WHOQOL-bref e a auto avaliação da QV.

Tabela 6 - Correlação de Pearson da variável resistência muscular localizada com os domínios retornados pelo instrumento WHOQOL-Bref

$\begin{array}{ccccc}\text { Domínio } & \text { Flexão de Braço } & \text { Abdominal } & \text { Flexibilidade } & \begin{array}{c}\text { Resistência } \\ \text { Aeróbica }\end{array} \\ \text { Domínio físico } & 0,268 & 0,259 & 0,100 & 0,281 \\ \text { Domínio psicológico } & 0,090 & 0,088 & 0,104 & -0,115 \\ \begin{array}{c}\text { Domínio relações } \\ \quad \text { sociais }\end{array} & 0,145 & 0,114 & 0,226 & -0,069 \\ \begin{array}{c}\text { Domínio ambiente } \\ \text { Auto avaliação da }\end{array} & 0,178 & 0,233 & -0,064 & 0,081 \\ \quad \text { QV } & 0,217 & 0,052 & 0,110 & 0,122 \\ \quad \text { Geral } & 0,222 & 0,215 & 0,094 & 0,074 \\ \text { Domínio físico } & 0,268 & 0,259 & 0,100 & 0,281\end{array}$

*Correlações significativas no nível $p>0,05$ quando valores forem maiores que 0,227 ou menores que $-0,227$. Correlações significativas no nível $p<0,05$ quando valores forem maiores que 0,296 ou menores que $-0,296$.

Fonte: Pesquisa de campo (2015).

Observa-se, no tocante aos testes pertinentes à RML, que houve correlação significativa para $p>0,05$ apenas entre o domínio físico e os testes de RML-1 $(0,268)$ e RML-2 $(0,259)$. Sendo assim, vale ressaltar que para a população 
investigada, quanto maior o nível de RML, maior a satisfação com o domínio físico, evidenciando uma característica de variáveis diretamente proporcionais.

Oliveira Junior et al. (2018), ao investigar a relação entre aptidão física, nível habitual de atividade física e QV, encontrou que a AFRS apresentou associação com o escore Psicológico da QV. Além disso, reportou que o nível habitual de atividade física apresentou correlação mais forte e significativa com a QV do que a AFRS. O nível habitual de atividade física também demonstrou exercer influência na QV no estudo de Maciel et al. (2016).

No entanto, quando se avaliou os resultados retornados pelo WHOQOL-Bref com o teste de AFRS, tal cenário não é tão evidente. A correlação apresentada entre os testes de RML e o domínio físico da QV não se tratar de uma casualidade. Barbanti (1979) afirma que resistência é a capacidade de efetuar um mesmo movimento resistindo à fadiga muscular pelo maior período de tempo possível. No domínio físico avaliado pelo instrumento WHOQOL-bref, a dor, a fadiga e a energia são variáveis consideradas pertinentes. Desta forma, um indivíduo que apresente uma maior resistência muscular localizada tende a sentir-se satisfeito em relação a estes quesitos.

Ainda em relação aos testes de RML-1 e RML-2, não foram encontradas correlações significativas para $p>0,01$ e $p>0,05$ com os domínios psicológico $(0,090 / 0,088)$, relações sociais $(0,145 / 0,114)$ e ambiente $(0,178 / 0,233)$. Também não foram significativas as correlações entre os testes de RML-1 e RML-2 com a auto avaliação da QV $(0,217 / 0,052)$ e com o domínio geral $(0,222 / 0,215)$.

Este cenário não parece se tratar de uma anormalidade. Aragão, Dantas e Dantas (2002), ao investigarem a resistência muscular localizada em idosos, não encontrou correlação significativa entre a satisfação com a QV e uma boa aptidão nos testes de resistência muscular localizada.

Quanto à flexibilidade, não foi possível identificar nenhuma correlação significativa para $p>0,01$ e $p>0,05$ entre $o$ teste de flexibilidade e os domínios (físico $-0,100$, psicológico 0,104 , relações sociais 0,226 , ambiente $-0,064)$. A correlação entre o domínio geral $(0,094)$ e a autoavaliação da QV $(0,110)$ também não foram significativas.

Pracidelli e Cabral (2011), ao investigarem o efeito do alongamento na flexibilidade e QV de mulheres jovens, constataram que o alongamento muscular, enquanto exercício físico, proporciona um aumento na satisfação dos componentes mentais e sociais da QV. Tal constatação permite o entendimento de que a flexibilidade, capacidade física aprimorada pelos exercícios de alongamento, poderia estar correlacionada com algum domínio da QV. No entanto, no presente estudo, não foi possível confirmar esta hipótese. A literatura não dispõe de evidências de possíveis correlações entre aspectos pertinentes aos domínios da QV avaliados pelo WHOQOL-bref e a flexibilidade enquanto capacidade física ligada a AFRS. Ainda assim, outros estudos podem encontrar cenário distinto ao exposto na presente investigação.

Por fim, no que concerne à resistência aeróbica, houve uma correlação significativa para $p>0,05$ entre os resultados do teste de Cooper e o domínio físico $(0,281)$. Isto permite inferir que, para o grupo investigado, quanto melhor a performance no teste de Cooper, maior a satisfação em relação ao domínio físico, indicando uma tendência de variáveis diretamente proporcionais.

Quanto aos demais domínios (psicológico -0,115, relações sociais -0,069 e ambiente 0,081 ), estes não apresentaram correlações significativas com o teste de Cooper para resistência aeróbica. Este cenário replicou-se na comparação dos 
resultados entre o teste de Cooper e a auto avaliação da $Q V(0,122)$ e o domínio geral $(0,074)$.

Amorim e Dantas (2002), ao investigarem os efeitos do exercício aeróbico na QV de idosos, inferiram que a prática de atividade física aeróbica contribui para melhorar a QV. Logo, pode-se supor que a capacidade aeróbica pode correlacionar-se com a QV. Porém, esta afirmativa confirmou-se, no presente estudo, apenas quanto ao domínio físico $(0,281)$.

A alta correlação encontrada entre a boa capacidade aeróbica e o domínio físico $(0,281)$ vai ao encontro do que a literatura científica relata a respeito dos benefícios da boa aptidão física para a saúde (ARAUJO; ARAUJO, 2000; CONTE, 2003). A ausência de correlação entre os resultados do teste de Cooper e os domínios psicológico $(-0,115)$, relações sociais $(-0,069)$ e ambiente $(0,081)$ pode ter ocorrido devido ao fato de que, neste teste, $94 \%$ dos indivíduos apresentaram um baixo desempenho. Isto, tal qual ocorreu em outros testes, dificulta a presença de correlações com variáveis em que as ligações não são conceitualmente próximas.

Em suma, verifica-se a presença de correlações significativas entre o nível de aptidão física das capacidades físicas relacionadas à saúde com o domínio físico da QV retornada pelo instrumento WHOQOL-bref. Isto sugere que um bom condicionamento físico destas variáveis pode aumentar a satisfação em relação às questões de bem-estar físico, que é um importante fator da QV global. No entanto, não foram encontradas correlações significativas entre as variáveis IMC, percentual de gordura, RML-1, RML-2, flexibilidade e capacidade aeróbica com os domínios psicológico, relações sociais, ambiente, geral e auto avaliação da QV. Dessa forma, para que se possam precisar conclusões no que tange a estas variáveis, a fim de atingir o estado da arte da temática proposta, faz-se necessário novas incursões acadêmicas com este foco.

\section{CONSIDERAÇÕES FINAIS}

Conceitualmente, a Saúde, a AFRS e a QV estão associadas. Consensualmente, o exercício físico regular é tido como um meio de prevenção doenças, melhora das capacidades físicas pertinentes à AFRS e, em sentido adicional, um meio de elevar os índices de QV.

No entanto, os resultados do presente estudo não apresentaram uma correlação significativa para os resultados dos testes das capacidades físicas da AFRS com os escores do domínio geral e de auto avaliação da QV retornados pelo instrumento WHOQOL-bref. Pondera-se ainda que este cenário se replica nas correlações entre os domínios psicológico, ambiente e relações sociais. Também não foram encontradas correlações significativas entre o domínio físico e o teste de Flexibilidade, além das medidas de IMC e percentual de gordura.

Tais resultados podem ter ocorrido em virtude da característica homogênea da amostra, bem como pelo fato de que outras variáveis, relacionadas ao domínio Relações sociais e Ambiente, influenciarem a QV e não estarem diretamente ligadas a AFRS.

A correlação significativa ocorreu entre os resultados dos testes de RML-1, RML-2 e resistência aeróbica com os resultados do domínio físico da QV retornado pelo instrumento WHOQOL-bref, indicando que quanto maiores forem os índices de RML-1, RML-2 e resistência aeróbica, melhor será a satisfação em relação ao domínio físico. 
Entre as limitações do presente estudo, estão a característica homogênea da amostra, em boa parte dos testes realizados, e, por consequência de tal homogeneidade, a configuração de um " $n$ " reduzido da referida amostra. Sugerese para estudos futuros pesquisas com ampliação do tamanho da amostra, com a utilização de testes físicos relacionados a aptidão física relacionada a saúde mais complexos, e com a utilização da regressão linear para análise da relação entre as variáveis.

Pondera-se que o presente estudo atingiu o objetivo de correlacionar os resultados dos testes físicos da AFRS com os escores da QV retornados pelo instrumento WHOQOL-bref em estudantes universitários. Ressalta-se que, quanto ao bem-estar físico, há uma tendência de que este seja influenciado pelo nível de AFRS do indivíduo.

Por fim, ressalta-se a existência de íntima relação entre variáveis relacionadas à AFRS e QV, realçando a necessidade e pertinência de programas de extensão que privilegiem estas em seus objetivos, tal qual o caso estudado na presente investigação. 


\title{
Interrelation between health-related physical fitness and domains of the quality of life
}

\begin{abstract}
This study aims to verify the correlation between aspects of health-related physical fitness with domains of the quality of life (QOL) of college students linked to UTFPR in Action project. The WHOQOL-bref instrument was applied for the evaluation of QOL. For physical fitness' measure we used Body Mass Index (BMI), fat percentage and the following tests: the sit and reach flexibility, Cooper, abdominals and push-ups per minute (RML). The results returned significant correlations between the RML and Cooper test data with the WHOQOL-bref physical domain scores. The results of the RML, Cooper, BMI and fat percentage tests with the WHOQOL-bref psychological, environmental, and social domains did not correlate significantly. We concluded that the higher the RML and aerobic resistance indexes, the greater the individual's score of physical domain of QOL. Such correlation did not occur in other scenarios investigated in this study.
\end{abstract}

KEYWORDS: Physical fitness; Health; Quality of life. 


\section{REFERÊNCIAS}

ADAMI, F. S.; FEIL, C. C.; DAL BOSCO, S. M. Estado nutricional relacionado a qualidade de vida de idosos. Revista brasileira de ciências do envelhecimento humano, Passo Fundo, v. 12, n. 1, p. 28-40, jul. 2015

AMORIM, F. S.; DANTAS, E. H. M. Efeitos do treinamento da capacidade aeróbica sobre a qualidade de vida e autonomia de idosos. Fitness \& Performance Journal, Rio de Janeiro, v. 1, n. 3, p. 47-55, mar./abr. 2002.

ARAGÃO, J. C. B.; DANTAS, E. H. M.; DANTAS, B. H. A. Efeitos da resistência muscular localizada visando à autonomia funcional e a qualidade de vida do idoso. Fitness \& Performance Journal, Rio de Janeiro, v. 1, n. 3, p. 29-37, mar./abr. 2002.

ARAÚJO, D. S. M. S.; ARAÚJO, C. G. S. Aptidão física, saúde e qualidade de vida relacionada à saúde em adultos. Revista brasileira de Medicina do Esporte, São Paulo, v. 6, n. 5, p. 194-203, set./out. 2000.

BARBANTI, V. Teoria e Prática do Treinamento Desportivo. 2. ed. São Paulo: Editora Edgard Blucher. 1979.

CHATTERJI, S.; BICKENBACH, J. Considerações sobre a qualidade de vida. In: FLECK, Marcelo Pio Almeida. et al. A avaliação de qualidade de vida: guia para profissionais da saúde. Porto Alegre: Artmed, 2008. p. 40-47.

CHACHAMOVICH, E.; FLECK, M. P. A. (2008). Desenvolvimento do instrumento WHOQOL-OLD. In: FLECK, Marcelo Pio Almeida. (Org.). A avaliação de qualidade de vida: guia para profissionais da saúde. Porto Alegre: Artmed, 102-111 p.

CASTRO, J. M. et al., Prevalência de sobrepeso e obesidade e os fatores de risco associados em adolescentes. Revista Brasileira de Obesidade, Nutrição e Emagrecimento, São Paulo, v. 12, n. 69, p. 84-93, jan./fev. 2018.

CONTE, $M$. et al. Interações entre $\mathrm{VO}^{2}$ Max, índice de massa corporal e flexibilidade. Revista Mackenzie de Educação Física e Esporte, São Paulo, v. 2, n. 2, p. 23-30, ago./out. 2003.

CRUZ, R. T. et al. Verificação do estado nutricional de estudantes do curso de nutrição das faculdades integradas de Bauru-SP com enfoque na Ortorexia. Revista Brasileira de Obesidade, Nutrição e Emagrecimento, São Paulo, v. 12, n. 76, p. 1119-1128, jan./dez. 2018.

DANCEY, C. P; REIDY, J. Estatística sem matemática para psicológica. 3 ed. Porto Alegre: Artmed, 2006.

US DEPARTAMENT OF HEALTH AND HUMAN SERVICES. Physical Activity and Health: A report of the Surgeon General. Pittsburgh: Superintendent of Documents, 1996.

FARQUHAR, M. Definitions of quality of life: a taxonomy. Journal of advanced nursing, Oxford, v. 22, n. 3, p. 502-508, sep. 1995. 
FERNANI, D. C. C. L. et al. Perfil antropométrico, nível de atividade física, grau de ansiedade e postura em universitários. Fisioterapia e Pesquisa, São Paulo, v. 24, n. 2, p. 191-197, jun. 2017. crossref

FLECK, M. P. A. et al. Desenvolvimento da versão em português do instrumento de avaliação de qualidade de vida da OMS (WHOQOL-100). Revista Brasileira de Psiquiatria, São Paulo, v. 21, n. 1, p. 19-28, jan./mar. 1999. crossref

FLECK, M. P. A. Problemas conceituais em qualidade de vida. In: FLECK, M. P. A. et al. (Org.). A avaliação de qualidade de vida: guia para profissionais da saúde. Porto Alegre: Artmed, 2008. p. 19-28.

GARCIA, C. P. Fundamentos teóricos de las capacidades físicas. Madrid: Vision Libros, 2009.

GARCIA, C. A. M. S.; MORETTO, M. C.; GUARIENTO, M. E. Estado nutricional e qualidade de vida em idosos. Revista da sociedade brasileira de clínica médica, São Paulo, v. 14, n. 1, p. 52-56, mai. 2016.

GLANER, M. F. Aptidão física relacionada à saúde de adolescentes rurais e urbanos em relação a critérios de referência. Revista Brasileira de Educação Física e Esporte, São Paulo, v. 19, n. 1, p. 13-24, jan./mar. 2005.

HOFFMAN, J. Norms for Fitness, performance and Health. New Zeland: Human Kinetics, 2006.

OLIVEIRA, H. F. R. et al. QVT-25: Construção e validação de um instrument de avaliação de Qualidade de Vida no Trabalho. Revista CPAQV, v. 9, n. 1, p. 1-15, jan. 2017.

OLIVEIRA JÚNIOR, A. C. M. et al. Padrões de aptidão física e qualidade de vida de bombeiros militares. Revista de Educação Física, v. 87, n. 1, p. 260-270, mar. 2018.

OMS. Obesety: Preventing and managing the global epidemic. Geneva: WHO Library Catalouguing, 2000.

MAZINI FILHO, M. L. et al. A prática de atividade física e o desempenho na aptidão física e saúde de discentes do curso noturno de educação física de uma instituição privada de uma cidade de minas gerais. Revista Brasileira de prescrição e fisiologia do exercício, São Paulo, v. 9, n. 53, p. 250-260, mai./jun. 2015.

MACIEL, E. S. Influência do nível de atividade física na percepção de qualidade de vida em comunidade universitária. Revista Brasileira de Qualidade de Vida, v. 8, n. 1, p. 42-56, jan./mar. 2016. crossref

PATRICK, D. A qualidade de vida pode ser medida? Como?. In: FLECK, Marcelo Pio Almeida et al. (Org.). A avaliação de qualidade de vida: guia para profissionais da saúde. Porto Alegre: Artmed, 2008. p. 29-39. 
PEDROSO, B. et al. Quality of life: a study based on the program UTFPR in action. The FIEP Bulletim, Foz do Iguaçu, v. 77, n. 1, p. 96-98, 2007.

PEDROSO, B. et al. Cálculo dos escores e estatística descritiva do WHOQOL-bref através do Microsoft Excel. Revista Brasileira de Qualidade de vida, Ponta Grossa, v, 2. n. 1, p. 31-36, 2010.

PEDROSO, B. Possibilidades e limites da avaliação da qualidade de vida: análise dos instrumentos WHOQOL e modelos clássicos de qualidade de vida no trabalho. 2013. 343f. Tese (Doutorado em Educação Física) - Faculdade de Educação Física, Universidade Estadual de Campinas, Campinas, 2013.

PEDROSO, B.; PILATTI, L. A. Guia de avaliação de Qualidade de vida e Qualidade de vida no trabalho. Editora UEPG: Ponta Grossa, 2012.

PRACIDELI, J.; CABRAL, C. M. N. Efeitos do alongamento na qualidade de vida e flexibilidade de mulheres jovens. ConScientiae Saúde, São Paulo, v. 10, n. 3, p. 539-542, 2011.

RODRIGUES, S. C.; ABOURIHAN, C. L. S.; YAMANE, R. Qualidade de vida e estado nutricional em homens idosos instituicionalizados. Cadernos da Escola de Saúde, Curitiba, v. 3, n. 1, p. 1-14, jan. 2010.

RIBEIRO, C. C. A. et al. Nível de flexibilidade obtida pelo teste de sentar e alcançar a partir de estudo realizado na Grande São Paulo. Revista Brasileira de Cineantropometria e Desempenho Humano, Florianópolis, v. 12, n. 6, p. 415421, dez./jul. 2010. crossref

TOSCANO, J. J. O.; OLIVEIRA, A. C. C. Qualidade de vida em idosos com distintos níveis de atividade física. Revista Brasileira de Medicina do Esporte, São Paulo, v. 15, n. 3, p. 169-173, mai./jun. 2009.
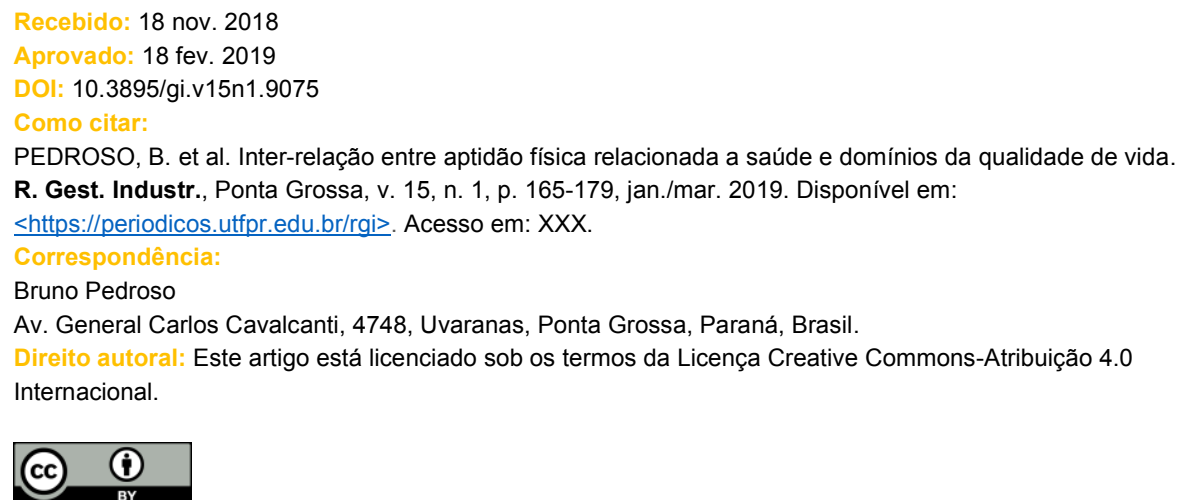